\title{
DINÂMICA DA DORMÊNCIA DE GEMAS DE VIDEIRA E QUIVIZEIRO EM REGIÃO DE BAIXA OCORRÊNCIA DE FRIO ${ }^{1}$
}

\author{
LUIZ ANTONIO BIASI ${ }^{2}$, RUY INACIO NEIVA DE CARVALHO ${ }^{3}$, FLÁVIO ZANETTE ${ }^{4}$
}

RESUMO - O objetivo desta pesquisa foi determinar a dinâmica da dormência de gemas de videira cv. Niagara Branca e de quivizeiro cv. Bruno, em região de baixa ocorrência de frio. Os experimentos foram realizados nos anos de 2007 e 2008, com coletas de ramos a cada duas semanas, em pomares localizados em Pinhais-PR. Na última coleta, um grupo adicional de ramos foi coletado e mantido em refrigerador, a temperatura de $4 \mathrm{a} 7^{\circ} \mathrm{C}$, por 1.440 horas. A avaliação da dormência foi realizada por meio do teste biológico de estacas de nós isolados em sala de crescimento, à temperatura de $25^{\circ} \mathrm{C}$ e fotoperíodo de 16 horas. Foi realizada a quantificação de horas de frio (HF) e de unidades de frio (UF) ocorridas na região. As duas espécies foram avaliadas como experimentos distintos. O delineamento experimental adotado foi o completamente casualizado, com doze tratamentos em 2007 e onze tratamentos em 2008, ambos com quatro repetições e dez estacas por unidade experimental. A endodormência de gemas de quivizeiro 'Bruno' instala-se a partir de abril e atinge maior intensidade do final de maio à segunda metade de junho. A saída da endodormência pode ocorrer naturalmente até o fim de agosto, mas com variações entre os anos. A endodormência de gemas de videira 'Niagara Branca' instala-se em abril e mantém-se intensa até maio. A saída natural da endodormência ocorre da metade de agosto ao início de setembro.

Termos para Indexação: Vitis labrusca, Actinidia deliciosa, brotação, endodormência.

\section{DORMANCY DYNAMICS OF GRAPE AND KIWI TREE BUDS IN A REGION OF LOW CHILLING OCCURRENCE}

\begin{abstract}
The objective of this work was to evaluate the dormancy dynamic of 'Niagara Branca' grape and 'Bruno' kiwi tree buds in a region of low chilling. The experiments were carried out in 2007 and 2008 through the cutting of branches every two weeks in orchards in Pinhais-PR. In the last date, an additional group of stems was collected and maintained in a refrigerator at a temperature of 4 to $7^{\circ} \mathrm{C}$ by 1440 hours. The evaluation of dormancy was observed by the biological test of single node cuttings into a growth room with temperature of $25^{\circ} \mathrm{C}$ and photoperiod of 16 hours. The chilling hours $(\mathrm{CH})$ and chilling units $(\mathrm{CU})$ were estimated from the region. The two species were analyzed individually. The entirely randomized experimental design was used with 12 treatments in 2007 and 11 treatments in 2008, both with four replications and ten cutting per plot. The bud endodormancy of 'Bruno' kiwi installed from April and reached higher intensity in the end of May to the second half of June. The end of endodormancy can naturally occur by the end of August, but with variations among years. The bud endodormancy of grapevine 'Niagara Branca' installed in April and remains strong until May. The end of natural endodormancy occurs half of August to early September. Index terms: Vitis labrusca, Actinidia deliciosa, budburst, endodormancy.
\end{abstract}

\footnotetext{
${ }^{1}$ (Trabalho 197-09). Recebido em:21-08-2009. Aceito para publicação em: 13-05-2010.

${ }^{2}$ Engenheiro Agrônomo, Dr., Professor Associado do Departamento de Fitotecnia e Fitossanitarismo. UFPR. Bolsista de Produtividade em Pesquisa do CNPq, biasi@ufpr.br.

${ }^{3}$ Engenheiro Agrônomo, Dr., Professor Titular do Centro de Ciências Agrárias e Ambientais da Pontifícia Universidade Católica do Paraná, Câmpus São José dos Pinhais. Bolsista de Produtividade em Pesquisa do CNPq, ruy.carvalho@pucpr.br.

${ }^{4}$ Engenheiro Agrônomo, Dr., Professor Titular do Departamento de Fitotecnia e Fitossanitarismo da UFPR, Bolsista de Produtividade em Pesquisa do CNPq, flazan@ufpr.br.
} 
A implantação de pomares de espécies frutíferas de clima temperado, em regiões subtropiciais e até mesmo tropicais, trouxe problemas de adaptação que não ocorriam nas regiões de origem, principalmente em relação à superação da dormência. O aquecimento global também é uma preocupação para as regiões tradicionais de fruticultura de clima temperado, pois tem-se verificado uma redução no acúmulo de horas de frio ao longo dos anos. Conforme uma previsão elaborada para a Califórnia, até o ano de 2100, o acúmulo de horas de frio reduzirá 40 horas por década e, no final do século XXI, o total acumulado será de apenas 500 horas por inverno, o que trará grandes impactos para a fruticultura local (BALDOCCHI; WONG, 2008).

As regiões distintas geograficamente apresentam também potencialidade distinta para o cultivo das espécies de clima temperado, conforme verificado por Botelho et al. (2006) para o Estado do Paraná, cujas regiões tradicionais no cultivo de plantas de clima temperado apresentaram, no período de 2000 a 2004, médias entre 402,4 (Palmas) a 161,8 (Curitiba) horas de frio igual ou abaixo de $7,2^{\circ} \mathrm{C}$.

$\mathrm{O}$ objetivo deste trabalho foi determinar a dinâmica da dormência de gemas de videira cv. Niagara Branca de quivizeiro cv. Bruno em região de baixa ocorrência de frio.

Os experimentos foram realizados com ramos coletados nos pomares de videira cv. Niagara Branca e de quivizeiro cv. Bruno (25 23,303’ sul, 4907,993' oeste e altitude de 930 m) da Estação Experimental do Canguiri da UFPR, localizada no município de Pinhais-PR. Os pomares foram manejados em sistema de produção orgânico e as plantas conduzidas em espaldeira. Para a avaliação do frio natural ocorrido, foram calculados o número de horas de frio $\left(\leq 7,2^{\circ} \mathrm{C}\right)$ e as unidades de frio ocorridas de acordo com o modelo de Utah original e Utah modificado, calculado pelo software Hora Frio do Departamento de Agrometeorologia da EPAGRI, com base nos dados climáticos fornecidos pelo Simepar (2008).

Para cada espécie, foram realizadas coletas a cada duas semanas, durante o período de 04 de abril a 05 de setembro de 2007 e 10 de abril a 29 de agosto de 2008, quando ocorreu o início de brotação das videiras a campo. Na última coleta, um grupo adicional de ramos foi coletado e mantido em refrigerador, à temperatura de 4 a $7^{\circ} \mathrm{C}$, por 1.440 horas.

A avaliação da dormência foi realizada por meio do teste biológico em sala de crescimento, com temperatura de $25^{\circ} \mathrm{C}$ e fotoperíodo de 16 horas. Foram confeccionadas estacas com $6 \mathrm{~cm}$ de comprimento, contendo apenas uma gema na porção supe- rior. Na videira, foram utilizadas as gemas situadas entre o segundo e o décimo nó, contados a partir da base dos ramos e, no quivizeiro, foram utilizadas as gemas situadas entre o quarto e o décimo quarto nó.

$\mathrm{O}$ delineamento experimental adotado foi o completamente casualizado, com doze tratamentos, no ano de 2007, e onze tratamentos, no ano de 2008. Todos os experimentos foram realizados com quatro repetições. Cada parcela experimental foi formada por dez estacas mantidas em vasos plásticos com vermiculita umedecida como substrato.

As estacas foram avaliadas individualmente a cada dois ou três dias, até um período máximo de 40 dias para a videira e 50 dias para o quivizeiro, de acordo com os estádios de crescimento da gema vegetativa "Ponta Verde" (PV) (aparecimento de modificações na coloração da gema, ficando esta com o ápice esverdeado) e "Gema Aberta" (Gab) (aparecimento de folhas abertas).

Com base nesses estádios, foram calculados o tempo médio para brotação (TMB), que representa o número médio de dias passados entre a instalação do experimento e a detecção do estádio PV; a taxa final de brotação (TF), que representa a porcentagem de estacas com gemas que atingiram PV; a taxa de brotações vigorosas (TBV), que representa a porcentagem de estacas com gemas que apresentaram o estádio PV e evoluíram até o estádio Gab [TBV $=(\%$ de estacas com gemas no estádio Gab) x 100/ $\mathrm{TF}$, e a velocidade de brotação (VB), que avalia a brotação das gemas em função do tempo para a brotação, dada pela equação $\mathrm{VB}=\Sigma\left(\mathrm{n}_{\mathrm{i}} / \mathrm{t}_{\mathrm{i}}\right)$ (gemas/ dia), em que: $n_{i}=$ número de gemas que atingiram o estádio PV no tempo " $i$ ", e $t_{i}=$ tempo após instalação do teste $(\mathrm{i}=1 \rightarrow 40$ ou 50$)$.

O município de Pinhais-PR, onde estavam localizados os pomares utilizados no experimento, apresentou baixo acúmulo de frio nos dois anos avaliados. O número de horas de frio (HF) abaixo de $7,2^{\circ} \mathrm{C}$, registrado em 2007, foi de $201 \mathrm{e}$, no ano de 2008, apenas 94 (Tabela 1). Para o quivizeiro, essa quantidade de frio é insuficiente para satisfazer sua exigência, que no caso da cultivar Bruno é considerada de 300 a 400 horas de frio (PETRI et al., 1996). Essa deficiência de frio ocasiona a falha da brotação das gemas laterais, e apenas a gema superior do ramo podado brota. Esse é um sintoma normalmente observado no pomar de quivizeiro em estudo. Já as videiras americanas, que possuem menor exigência em frio e também pelo fato de receberem podas muito curtas, não apresentam problemas de brotação na região em estudo. Pelo cálculo das unidades de frio (UF), pelo método original de Utah, observou-se um somatório negativo nos dois anos avaliados (Tabela 1), demons- 
trando que os períodos de outono e inverno avaliados apresentaram poucas horas com temperaturas entre 1,5 e $12,4^{\circ} \mathrm{C}$, que é a faixa considerada pelo método como a ideal para o acúmulo de unidades de frio e também muitas horas com temperaturas acima de $16^{\circ}$ $\mathrm{C}$, que anulam o frio acumulado. Com o método de Utah modificado, calculado pelo programa HoraFrio, que limita bastante o efeito negativo das temperaturas elevadas, observou-se acúmulo positivo de unidades de frio, porém semelhantes às horas de frio calculadas. As unidades de frio acumuladas por esse método foram de 223,5 UF em 2007 e 93 UF em 2008. Esses métodos de quantificação do frio são questionáveis, pois o simples somatório de HF não considera as flutuações climáticas e o momento fisiologicamente correto em que as gemas acumulam frio para superar a endodormência. O método de Utah é adequado para climas temperados, tornando-se limitado em climas subtropicais (DENNIS JÚNIOR, 2003).

Na primeira e na quarta coletas de 2007 e nas quatro primeiras coletas em 2008, não houve brotação de gemas do quivizeiro dentro do período de 50 dias avaliado. Essa resposta pode estar relacionada com o efeito da paradormência devido à presença de folhas e frutos nas plantas de onde foram retirados os ramos. A paradormência refere-se à influência de outro órgão do vegetal sobre a gema (LANG et al., 1987) e cessa quando aquele é retirado. No entanto, mesmo após a colheita dos frutos e a queda de folhas, o TMB observado foi alto, sendo superior a 35 dias em 2007 e 42,7 dias em 2008 (Tabela 2), que deve ter ocorrido pelo efeito da endodormência já instalada nas gemas nessa época. Em 2007, o TMB manteve-se alto e superior às demais coletas até a data de 13 de junho, que apresentou TMB de 37 dias. Em 2008, o TMB foi alto e superior até 19 de junho, com 42,7 dias (Tabela 2). Nessas datas com alto TMB, também se observaram baixas TF, VB e TBV. A taxa final de brotação (TF) variou entre 15 a 45\% em 2007 e 22,5 a $27,5 \%$ em 2008, enquanto a taxa de brotações vigorosas (TBV) variou de 0 a 46,2\% em 2007 e 8,3 a $16,7 \%$ em 2008 , nos períodos de endodormência mais profunda, o que demonstra pouca capacidade de brotação das gemas, mesmo em condições favoráveis de crescimento.

$\mathrm{Na}$ última coleta de quivizeiro realizada nos dois anos, ocorreu o menor TMB do período, sendo 14,7 dias em 2007 e 11,2 dias em 2008, valores superiores aos observados após o tratamento com 1.440 horas de frio adicional, que foi de 6,5 dias em 2007 e 7,2 dias em 2008 (Tabela 2). Isso comprova que o acúmulo de frio durante os dois anos foi insuficiente para superar a dormência do quivizeiro. O tratamento com frio adicional também aumentou significativa- mente a VB.

Para a videira, apenas as gemas coletadas em 04 de abril de 2007 não brotaram. O TMB, em 2007, aumentou até 30 de maio, quando atingiu o maior valor aos 30,5 dias. A partir dessa data, houve redução gradativa do TMB até a estabilização em 22 de agosto, revelando que as gemas já estavam prontas para brotar. Em 2008, o TMB já iniciou alto na primeira coleta de 10 de abril e permaneceu elevado até 08 de maio. A partir dessa data, também ocorreu redução gradual até a estabilização em 14 de agosto (Tabela 2). A entrada precoce em dormência das gemas de videira não foi associada a um efeito de paradormência, pois desde as primeiras coletas as plantas não possuíam folhas. Provavelmente, as gemas já se encontravam em endodormência, mas não induzidas pelo frio, pois até 02 de maio de 2007 e 24 de abril de 2008 não ocorreu nenhuma hora de frio abaixo de $7,2^{\circ} \mathrm{C}$ ou unidades de frio calculadas pelo método de Utah (Tabela 1). Possivelmente, a entrada em endodormência ocorreu pela mudança no fotoperíodo. A entrada em dormência na espécie Vitis riparia foi induzida após 4 semanas de exposição a fotoperíodo com dias curtos, de 8 horas de luz (WAKE; FENNELL, 2000).

A taxa final de brotação (TF) e a taxa de brotações vigorosas (TBV) somente apresentaram diferença significativa no ano de 2007, nas datas de dormência mais profunda, possivelmente pela maior irregularidade das temperaturas naquele ano. O TMB das últimas coletas realizadas em 2007 e 2008 não diferiu significativamente das estacas que receberam frio adicional por 1.440 horas, revelando que as gemas já estavam prontas para brotar. Esse fato também foi observado no campo, pois nessas coletas já havia gemas brotando. Esse comportamento indica que a exigência em frio da videira 'Niagara Branca' não é alta, pois a ocorrência de apenas 94 HF em 2008 foi suficiente para sua brotação.

A endodormência de gemas de quivizeiro 'Bruno' instala-se a partir de abril e atinge maior intensidade do final de maio à segunda metade de junho. A saída da endodormência pode ocorrer naturalmente até fim de agosto, mas com variações entre os anos.

A endodormência de gemas de videira 'Niagara Branca' instala-se em abril e mantém-se intensa até maio. A saída natural da endodormência ocorre da metade de agosto ao início de setembro. 
TABELA 1 - Horas de frio abaixo de $7,2^{\circ} \mathrm{C}$, unidades de frio (UF) calculadas pelos modelos Utah original e Utah modificado e estimado pelo Programa HoraFrio, registradas pela Estação Meteorológica do SIMEPAR, localizada em Pinhais-PR, em cada data de coleta em 2007 e 2008.

\begin{tabular}{ccccccc}
\hline Períodos & \multicolumn{2}{c}{ Horas de frio $\leq 7,2^{\circ} \mathrm{C}$} & \multicolumn{2}{c}{ UF (Modelo Utah) } & UF (Modelo Utah modificado) \\
\cline { 2 - 7 } Ano 2007 & $\begin{array}{c}\text { Entre as } \\
\text { datas }\end{array}$ & Acumuladas & $\begin{array}{c}\text { Entre as } \\
\text { datas }\end{array}$ & Acumuladas & $\begin{array}{c}\text { Entre as } \\
\text { datas }\end{array}$ & Acumuladas \\
\hline 01-04 a 03-04 & 0 & 0 & $-72,0$ & -72 & $-47,5$ & 0,0 \\
04-04 a 17-04 & 0 & 0 & $-223,0$ & $-295,0$ & $-223,0$ & 0,0 \\
18-04 a 01-05 & 0 & 0 & $-189,5$ & $-484,5$ & $-191,0$ & 0,0 \\
02-05 a 15-05 & 8 & 8 & $-72,0$ & $-556,5$ & $-78,0$ & 8,5 \\
16-05 a 29-05 & 36 & 44 & 13,0 & $-543,5$ & 22,0 & 76,5 \\
30-05 a 12-06 & 42 & 86 & $-12,5$ & $-556,0$ & $-55,0$ & 92,5 \\
13-06 a 26-06 & 0 & 86 & $-91,0$ & $-647,0$ & $-86,5$ & 108,0 \\
27-06 a 10-07 & 6 & 92 & $-63,5$ & $-710,5$ & $-70,0$ & 73,0 \\
11-07 a 24-07 & 30 & 122 & 60,5 & $-650,0$ & 90,5 & 163,5 \\
25-07 a 05-08 & 74 & 196 & 104,0 & $-546,0$ & 108,5 & 272,0 \\
06-08 a 21-08 & 0 & 196 & $-15,5$ & $-561,5$ & $-16,0$ & 256,0 \\
22-08 a 05-09 & 5 & 201 & $-29,0$ & $-590,5$ & $-31,5$ & 223,5 \\
\hline & & & & & \\
Ano 2008 & Entre as & Acumuladas & Entre as & Acumuladas & Entre as & Acumuladas \\
\hline 01-04 a 09-04 & 0 & 0 & $-105,0$ & -105 & $-99,5$ & 0,0 \\
10-04 a 23-04 & 0 & 0 & $-144,0$ & $-249,0$ & $-142,0$ & 0,0 \\
24-04 a 07-05 & 6 & 6 & $-32,5$ & $-281,5$ & $-23,0$ & 50,0 \\
08-05 a 20-05 & 1 & 7 & 12,5 & $-269,0$ & 9,0 & 59,0 \\
21-05 a 03-06 & 8 & 15 & $-49,0$ & $-318,0$ & $-48,5$ & 103,5 \\
04-06 a 18-06 & 53 & 68 & $-20,0$ & $-338,0$ & $-7,0$ & 121,5 \\
19-06 a 02-07 & 12 & 80 & 25,5 & $-312,5$ & 27,0 & 155,0 \\
03-07 a 16-07 & 14 & 94 & 9,0 & $-303,5$ & $-13,0$ & 170,5 \\
17-07 a 30-07 & 0 & 94 & $-59,0$ & $-362,5$ & $-56,5$ & 137,5 \\
31-07 a 13-08 & 0 & 94 & 17,5 & $-345,0$ & 4,0 & 159,5 \\
14-08 a 29-08 & 0 & 94 & $-107,5$ & $-452,5$ & $-106,0$ & 93,0 \\
\hline
\end{tabular}


TABELA 2 - Tempo médio para brotação (TMB), taxa final de brotação (TF), velocidade de brotação (VB) e taxa de brotações vigorosas (TBV) no teste biológico de estacas de nós isolados de quivizeiro 'Bruno' e de videira 'Niagara Branca' coletadas em Pinhais-PR, 2007 e 2008.

\begin{tabular}{|c|c|c|c|c|c|c|c|c|}
\hline \multirow{2}{*}{$\begin{array}{c}\text { Datas de coleta } \\
2007\end{array}$} & \multicolumn{4}{|c|}{ Quivizeiro } & \multicolumn{4}{|c|}{ Videira } \\
\hline & $\begin{array}{l}\mathrm{TMB}^{* *} \\
\text { (dias) }\end{array}$ & $\begin{array}{l}\mathrm{TF}^{* *} \\
(\%)\end{array}$ & $\begin{array}{c}\text { VB** } \\
\left(\text { gemas dia }^{-1}\right)\end{array}$ & $\begin{array}{c}\mathrm{TBV}^{* *} \\
(\%)\end{array}$ & $\begin{array}{l}\mathrm{TMB}^{* *} \\
\text { (dias) }\end{array}$ & $\begin{array}{l}\mathrm{TF}^{* *} \\
(\%)\end{array}$ & $\begin{array}{c}\text { VB** } \\
\left(\text { gemas dia }^{-1}\right)\end{array}$ & $\begin{array}{c}\mathrm{TBV}^{* *} \\
(\%)\end{array}$ \\
\hline 04-04 & $\mathrm{ND}^{* * * *}$ & 0,0 & 0,0 & ND & $\mathrm{ND}^{* * *}$ & 0,0 & 0,0 & ND \\
\hline $18-04$ & $35,0 \mathrm{ab}$ & $22,5 \mathrm{ef}$ & $0,06 \mathrm{~cd}$ & $8,3 \mathrm{~cd}$ & $15,8 \mathrm{~cd}$ & $100,0 \mathrm{a}$ & $0,64 \mathrm{~cd}$ & $100,0 \mathrm{a}$ \\
\hline $02-05$ & $38,0 \mathrm{a}$ & 30,0def & $0,08 \mathrm{~cd}$ & $12,5 \mathrm{bcd}$ & $27,3 \mathrm{ab}$ & $90,0 \mathrm{a}$ & $0,34 \mathrm{f}$ & $91,8 \mathrm{ab}$ \\
\hline $16-05$ & ND & 0,0 & 0,0 & ND & $23,8 b$ & $50,0 \mathrm{~b}$ & $0,21 \mathrm{f}$ & $100,0 \mathrm{a}$ \\
\hline $30-05$ & $35,0 \mathrm{ab}$ & $45,0 \mathrm{bcdef}$ & $0,13 \mathrm{~cd}$ & $46,2 \mathrm{abcd}$ & $30,5 \mathrm{a}$ & $77,5 \mathrm{a}$ & $0,26 f$ & $75,8 \mathrm{c}$ \\
\hline $13-06$ & $37,0 \mathrm{a}$ & $15,0 \mathrm{f}$ & $0,04 d$ & $0,0 \mathrm{~d}$ & $25,5 b$ & $97,5 \mathrm{a}$ & $0,39 \mathrm{ef}$ & $97,5 \mathrm{a}$ \\
\hline $27-06$ & $29,1 \mathrm{~cd}$ & $25,0 \mathrm{def}$ & $0,12 \mathrm{~cd}$ & $27,1 \mathrm{abcd}$ & $26,5 b$ & $82,5 a$ & $0,31 \mathrm{f}$ & $97,2 \mathrm{a}$ \\
\hline $11-07$ & $22,8 \mathrm{e}$ & $57,5 \mathrm{dcd}$ & $0,25 b c$ & $60,0 \mathrm{ab}$ & $17,1 \mathrm{c}$ & $100,0 \mathrm{a}$ & $0,60 \mathrm{de}$ & $100,0 \mathrm{a}$ \\
\hline $25-07$ & $31,5 b c$ & 52,5 bcde & $0,17 \mathrm{~cd}$ & $73,1 \mathrm{a}$ & $12,7 \mathrm{~d}$ & $97,5 \mathrm{a}$ & $0,86 \mathrm{c}$ & $100,0 \mathrm{a}$ \\
\hline 06-08 & $29,9 \mathrm{bcd}$ & $75,0 \mathrm{ab}$ & $0,26 b c$ & $67,4 a$ & $13,1 d$ & $97,5 \mathrm{a}$ & $0,76 \mathrm{~cd}$ & $100,0 \mathrm{a}$ \\
\hline $22-08$ & $25,2 \mathrm{de}$ & $40,0 \mathrm{cdef}$ & $0,16 \mathrm{~cd}$ & $50,0 \mathrm{abc}$ & $6,7 \mathrm{e}$ & $100,0 \mathrm{a}$ & $1,55 b$ & $100,0 \mathrm{a}$ \\
\hline 05-09 & $14,7 f$ & $67,5 \mathrm{abc}$ & $0,46 b$ & $63,9 a$ & $5,6 \mathrm{ef}$ & $97,5 \mathrm{a}$ & $1,79 b$ & $82,2 \mathrm{bc}$ \\
\hline 05-09+Frio* & $6,5 \mathrm{~g}$ & $92,5 \mathrm{a}$ & $1,70 \mathrm{a}$ & $64,5 \mathrm{a}$ & $2,1 \mathrm{f}$ & $100,0 \mathrm{a}$ & $4,79 a$ & $100,0 \mathrm{a}$ \\
\hline CV $(\%)$ & 8,02 & 28,79 & 27,52 & 45,94 & 8,84 & 10,46 & 9,27 & 5,76 \\
\hline \multicolumn{9}{|l|}{2008} \\
\hline $10-04$ & $\mathrm{ND}^{* * *}$ & 0,0 & 0,0 & ND & $23,8 \mathrm{a}$ & $77,5 \mathrm{a}$ & $0,35 \mathrm{f}$ & $88,2 \mathrm{a}$ \\
\hline 24-04 & ND & 0,0 & 0,0 & ND & $20,6 a b c$ & $90,0 \mathrm{a}$ & $0,45 \mathrm{ef}$ & $91,2 \mathrm{a}$ \\
\hline 08-05 & ND & 0,0 & 0,0 & ND & $21,8 \mathrm{ab}$ & $95,0 \mathrm{a}$ & $0,45 \mathrm{ef}$ & $97,2 \mathrm{a}$ \\
\hline $21-05$ & ND & 0,0 & 0,0 & ND & $18,8 \mathrm{bc}$ & $95,0 \mathrm{a}$ & $0,52 \mathrm{ef}$ & $96,8 \mathrm{a}$ \\
\hline 04-06 & $44,1 \mathrm{a}$ & $22,5 \mathrm{~d}$ & $0,05 \mathrm{~d}$ & $16,7 \mathrm{~cd}$ & $17,1 \mathrm{~cd}$ & $100,0 \mathrm{a}$ & $0,60 \mathrm{ef}$ & $100,0 \mathrm{a}$ \\
\hline $19-06$ & $42,7 \mathrm{a}$ & $27,5 \mathrm{~d}$ & $0,06 \mathrm{~d}$ & $8,33 d$ & $19,0 \mathrm{bc}$ & $90,0 \mathrm{a}$ & $0,49 \mathrm{ef}$ & $96,4 \mathrm{a}$ \\
\hline 03-07 & $35,3 b$ & $50,0 \mathrm{c}$ & $0,14 d$ & $45,0 \mathrm{bcd}$ & $14,6 \mathrm{~d}$ & $97,5 \mathrm{a}$ & $0,69 \mathrm{ef}$ & $100,0 \mathrm{a}$ \\
\hline $17-07$ & $28,0 \mathrm{c}$ & $32,5 \mathrm{~cd}$ & $0,12 d$ & $60,8 \mathrm{abc}$ & $13,3 d$ & $100,0 \mathrm{a}$ & $0,77 \mathrm{e}$ & $100,0 \mathrm{a}$ \\
\hline $31-07$ & $22,7 d$ & $40,0 \mathrm{~cd}$ & $0,18 \mathrm{~d}$ & $62,5 \mathrm{abc}$ & $8,8 \mathrm{e}$ & $100,0 \mathrm{a}$ & $1,35 \mathrm{~d}$ & $100,0 \mathrm{a}$ \\
\hline $14-08$ & $20,5 d$ & $75,0 \mathrm{~b}$ & $0,38 \mathrm{c}$ & $80,4 a b$ & $4,9 \mathrm{ef}$ & $100,0 \mathrm{a}$ & $2,18 \mathrm{c}$ & $100,0 \mathrm{a}$ \\
\hline $29-08$ & $11,2 \mathrm{e}$ & $95,0 \mathrm{a}$ & $0,89 b$ & $100,0 \mathrm{a}$ & $3,8 \mathrm{f}$ & $100,0 \mathrm{a}$ & $3,01 \mathrm{~b}$ & $100,0 \mathrm{a}$ \\
\hline 29-08+Frio* & $7,2 \mathrm{e}$ & $100,0 \mathrm{a}$ & $1,50 \mathrm{a}$ & $100,0 \mathrm{a}$ & $2,3 f$ & $100,0 \mathrm{a}$ & $4,70 \mathrm{a}$ & $100,0 \mathrm{a}$ \\
\hline CV (\%) & 8,29 & 13,93 & 13,83 & 36,06 & 11,38 & 10,11 & 10,71 & 6,26 \\
\hline
\end{tabular}

*Tratamento com 1.440 horas de frio de 4 a $7^{\circ} \mathrm{C}$.

**Médias seguidas por letras distintas nas colunas diferem entre si, pelo teste de Tukey, ao nível de significância de 5\%.

*** Não determinado. Nenhuma gema brotou até o término das avaliações e essas datas não foram consideradas na análise estatística 


\section{REFERÊNCIAS}

BALDOCCHI, D.; WONG, S. Accumulated winter chill is decreasing in the fruit growing regions of Califórnia. Climatic Change, Dordrecht, v. 87, n.1, p. 153-166, 2008. Suplemento.

BOTELHO, R. V.; AYUB, R. A.; MÜLLER, M. M. L. Somatória de horas de frio e de unidades de frio em diferentes regiões do Estado do Paraná. Scientia Agraria, Curitiba, v. 7, n. 1-2, p. 89-96, 2006.

DENNIS JUNIOR, F.G. Problems in standardizing methods for evaluating the chilling requirements for the breaking of dormancy in buds of woody plants. HortScience, Alexandria, v. 38, n. 3, p. 347-350, 2003.
LANG, G. A.; EARLY, J. D.; MARTIN, G. C.; DARNELL, R. L. Endo-, para- and ecodormancy: physiological terminology and classification for dormancy research. Hortscience, Alexandria, v.22, p.371-178, 1987.

PETRI, J. L.; PALLADINI, L. A.; SCHUCK, E.; DUCROQUET, J. P.; MATOS, C. S.; POLA, A. C. Dormência e indução da brotação de fruteiras de clima temperado. Florianópolis: Epagri, 1996. 110p. (Boletim Técnico, 75).

SIMEPAR. Temperatura média horária. Curitiba, 2008. Arquivo Eletrônico Word for Windows.

WAKE, C.M.F.; FENNELL, A. Morphological, physiological and dormancy responses of three Vitis genotypes to short photoperiod. Physiologia Plantarum, Copenhagen, v. 109, n. 2, p. 203-210, 2000. 